\title{
ATUAÇÃO DO MÉDICO VETERINÁRIO NA PREVENÇÃO DE AGRAVOS A SAÚDE DO TRABALHADOR
}

\author{
VETERINARIAN PERFORMANCE IN THE PREVENTION OF HARMS TO THE \\ WORKER'S HEALTH
}

\author{
M. J. L. SICONELLI ${ }^{1 *}$, A. M. TEDESCO ${ }^{2}$, N. A. ASSIS ${ }^{3}$, K. P. BÜRGER ${ }^{4}$
}

\section{RESUMO}

A Vigilância em Saúde, componente estruturante do SUS, está alicerçada sobre 4 pilares: Vigilância Epidemiológica, Sanitária, Ambiental e Saúde do Trabalhador. É na Saúde do Trabalhador que se insere a Comissão Interna de Prevenção de Acidentes (CIPA) e o médico veterinário, profissional habilitado para compor e atuar em tais grupos de trabalho. Objetivou-se realizar o diagnóstico de situação do Hospital Veterinário "Governador Laudo Natel" (HV) da FCAV/ UNESP, Jaboticabal, sobre a saúde dos seus funcionários. Averiguando legalmente, segundo o Decreto-Lei $\mathrm{n}^{\circ} 5.452$, a NR 5 e NR 7 que visam defender e monitorar a saúde do trabalhador. Participaram 33 funcionários do HV, que a partir de um inquérito sobre a Saúde do Trabalhador, identificou o grau de entendimento de cada trabalhador sobre seus direitos, conhecimentos específicos no exercício da profissão na área da saúde e a preocupação da instituição empregatícia com a saúde dos trabalhadores. Além do inquérito também foi realizado o levantamento sorológico dos funcionários para brucelose, doença crônica e de sintomas inespecíficos, pelo teste de triagem do Antígeno Acidificado Tamponado (AAT), este preconizado dentro do Programa Nacional de Controle e Erradicação de Brucelose e Tuberculose, Ministério da Agricultura, Pecuária e Abastecimento (PNCEBT/ MAPA), e também utilizado como triagem em Medicina Humana. Verificouse falhas na difusão e conscientização do trabalhador. Situação que pode aumentar a chance de acidentes, resultando na aquisição de enfermidades como a brucelose, além de outras. Pelo AAT não houve amostras positivas, não havendo a necessidade de realizar um teste confirmatório. Mesmo com a CIPA estabelecida na UNESP, observa-se que a mesma não está presente no HV. Existe a necessidade de implementar um grupo que tenha conhecimento da realidade do local, trabalhando junto aos funcionários para mitigar riscos e mostrar a importância dos próprios funcionários na prevenção de agravos a própria saúde.

\section{PALAVRAS-CHAVES: INQUÉRITO. BRUCELOSE. AAT. VIGILÂNCIA EM SAÚDE.}

AGRADECIMENTOS: Laboratório de exames sorológicos de leptospirose e brucelose.

ÁREA TEMÁTICA: Saúde Pública.

\footnotetext{
${ }^{1}$ Residente em Medicina Veterinária Preventiva do Programa de Residência em Área Profissional da Saúde Medicina Veterinária e Saúde da Universidade Estadual Paulista "Júlio de Mesquita Filho", Faculdade de Ciências Agrárias e Veterinárias - FCAV. E-mail: marcio_siconelli@outlook.com

Médica Veterinária, graduada pela Universidade Estadual Paulista "Júlio de Mesquita Filho", Faculdade de Ciências Agrárias e Veterinárias - FCAV

${ }^{2}$ Médica Veterinária, graduada pela Universidade Estadual Paulista "Júlio de Mesquita Filho", Faculdade de Ciências Agrárias e Veterinárias - FCAV

${ }^{3}$ Assistente de Suporte Acadêmico II do Laboratório de Exames Sorológicos de Leptospirose e Brucelose da Universidade Estadual Paulista "Júlio de Mesquita Filho", Faculdade de Ciências Agrárias e Veterinárias - FCAV

${ }^{4}$ Docente do Departamento de Medicinava Veterinária Preventiva e Reprodução Animal da Universidade Estadual Paulista "Júlio de Mesquita Filho", Faculdade de Ciências Agrárias e Veterinárias - FCAV
} 